\title{
Hot Zero Power Reactor Calculations Using the Insilico Code ${ }^{\text {स }}$
}

\author{
Steven P. Hamilton ${ }^{\mathrm{a}, 1, *}$, Thomas M. Evans ${ }^{\mathrm{a}, 1}$, Gregory G. Davidson ${ }^{\mathrm{a}, 1}$, \\ Seth R. Johnson ${ }^{\mathrm{a}, 1}$, Tara M. Pandya ${ }^{\mathrm{a}, 1}$, Andrew T. Godfrey ${ }^{\mathrm{a}, 2}$ \\ ${ }^{a}$ Oak Ridge National Laboratory, 1 Bethel Valley Rd., Oak Ridge, TN 37831 U.S.A.
}

\begin{abstract}
In this paper we describe the reactor physics simulation capabilities of the Insilico code. A description of the various capabilities of the code is provided, including detailed discussion of the geometry, meshing, cross section processing, and neutron transport options. Numerical results demonstrate that Insilico using an $\mathrm{SP}_{\mathrm{N}}$ solver with pin-homogenized cross section generation is capable of delivering highly accurate full-core simulation of various pressurized water reactor problems. Comparison to both Monte Carlo calculations and measured plant data is provided.
\end{abstract}

Keywords: radiation transport, nuclear reactor physics, eigenvalue solvers, nuclear cross sections

\footnotetext{
Notice: This manuscript has been authored by UT-Battelle, LLC, under contract DE-AC05-00OR22725 with the U.S. Department of Energy. The United States Government retains and the publisher, by accepting the article for publication, acknowledges that the United States Government retains a non-exclusive, paid-up, irrevocable, world-wide license to publish or reproduce the published form of this manuscript, or allow others to do so, for United States Government purposes.

*Corresponding Author

Email addresses: hamiltonsp@ornl.gov (Steven P. Hamilton), evanstm@ornl.gov (Thomas M. Evans), davidsongg@ornl.gov (Gregory G. Davidson), johnsonsr@ornl.gov (Seth R. Johnson), pandyatm@ornl.gov (Tara M. Pandya), godfreyat@ornl.gov (Andrew T. Godfrey)

${ }^{1}$ Radiation Transport Group, Reactor and Nuclear Systems Division

${ }^{2}$ Reactor Physics Group, Reactor and Nuclear Systems Division
} 


\section{Introduction}

Accurate characterization of the neutronic behavior of an operating nuclear reactor plays a vital role in reactor design and analysis. Because reactor analyses often require numerous calculations to compute quantities of interest (e.g., control rod worth, differential boron worth, and reactivity coefficients), having tools capable of producing sufficiently accurate results with moderate computational requirements is imperative.

In this work, we introduce the Insilico code, a new neutronics package targeted at light water reactor problems. Insilico operates on a simple input specification that is optimized for pressurized water reactor (PWR) configurations, allowing modeling of common reactor features with minimal effort. Insilico is capable of using both deterministic radiation transport via the Denovo [1] code and Monte Carlo transport via the Shift [2] code. Within Denovo, both discrete ordinates $\left(\mathrm{S}_{\mathrm{N}}\right)$ and simplified spherical harmonics $\left(\mathrm{SP}_{\mathrm{N}}\right)$ angular approximations are available. Automated cross section processing is performed for the deterministic methods using the SCALE package [3], with support for either pin-resolved or spatially homogenized models.

The high computational expense of performing pin-resolved $\mathrm{S}_{\mathrm{N}}$ calculations has led to a focus on using coarse-mesh $\mathrm{SP}_{\mathrm{N}}$ with spatially homogenized cross sections as the primary deterministic approach in Insilico. Numerical experiments on a wide range of PWR configurations, including models of the initial criticality at Unit 1 of the Watts Bar nuclear plant and the Westinghouse AP $100{ }^{\circledR}$ design, indicate that a high level of accuracy is achievable with this approach.

The rest of the paper is organized as follows. In $\S 2$, a discussion of the geometry and meshing capabilities of Insilico is provided, along with a description of its input file format. In $\S 3$, the cross section capabilities of Insilico are presented. Deterministic radiation transport solution approaches employed by Insilico are described in $\S 4$. Results are shown in $\S 5$ and some concluding remarks are presented in $\S 6$.

\section{Problem Initialization and Meshing}

Insilico initializes a problem by reading a specification that defines all of the materials and geometric layout of the core. This information is processed upon input into an in-memory metadata model that is a realization of the physical core model. This metadata model consists of axial arrays of pin cells. 

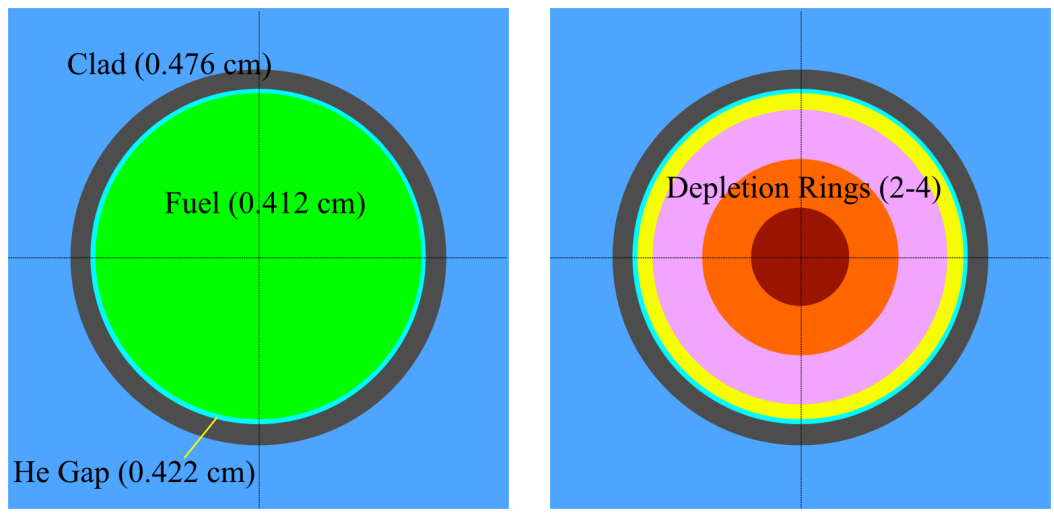

Figure 1: Metadata model examples of a clean and depleted fuel pin.

All relevant features in the core are modeled as pin cell variants; examples are shown in Fig. 1. Most of the neutronically important features of a PWR reactor core are included in the pin cell models, including nozzles, pin caps, core plates, and spacer grids. See Table 1 for information on how reactor core features are transformed to pin cells.

The simplifications described in Table 1 are necessary to convert all modeled reactor components to pin cells and are acceptable even for highresolution PWR modeling. Using this model, Insilico constructs and initializes a computational grid with material data as described later in this section. The metadata model also provides the material input for cross section processing, the methods for which are described in $\S 3$.

All of the deterministic transport methods used in Insilico are implemented for regular Cartesian grids. Although Cartesian mesh methods suffer from some drawbacks associated with resolving geometric features, the gains in computational efficiency often outweigh such issues and have thus been the focus of many modern radiation transport solvers [4-6]. Furthermore, it was shown in Ref. [7] that accurate within-pin distributions can be obtained from Cartesian mesh solutions using polynomial reconstruction approaches, an idea that has been the basis for multiple multiphysics coupling demonstrations $[8,9]$. Insilico provides two meshing options, pin-resolved and pin-homogenized, as illustrated in Figs. 3. Both of these meshing techniques overlay an $N \times M$ mesh on the pin cells. For pin-homogenized grids, each mesh cell within a given pin cell uses the same cross sections. The process for calculating the 
Table 1: Reactor component to pin cell conversion methodology

\begin{tabular}{cl}
\hline Model Component & \multicolumn{1}{c}{ Pin Cell Conversion Method } \\
\hline Axial reflectors & $\begin{array}{l}\text { Converted to homogeneous pin cells of reflector } \\
\text { material mixed with moderator at user-specified } \\
\text { weight fraction }\end{array}$ \\
\hline Core plates & $\begin{array}{l}\text { Converted to homogeneous pin cells of plate mate- } \\
\text { rial mixed with moderator at user-specified weight } \\
\text { fraction }\end{array}$ \\
\hline Assembly nozzles & $\begin{array}{l}\text { Converted to homogeneous pin cells of nozzle ma- } \\
\text { terial mixed with moderator }\end{array}$ \\
\hline Spacer grids & $\begin{array}{l}\text { Mixed into the pin cell moderator such that grid } \\
\text { mass is preserved }\end{array}$ \\
\hline Pin caps & $\begin{array}{l}\text { Modeled directly as a pin cell with a single cylinder } \\
\text { of clad material surrounded by moderator }\end{array}$ \\
\hline Plenum & $\begin{array}{l}\text { Modeled as two concentric cylinders of plenum gas } \\
\text { and clad surrounded by moderator }\end{array}$ \\
\hline Fuel cells & $\begin{array}{l}\text { Modeled as concentric cylinders of fuel, gap, and } \\
\text { clad surrounded by moderator (see Figure 1) }\end{array}$ \\
\hline Guide tubes & $\begin{array}{l}\text { Modeled as two concentric cylinders of moderator } \\
\text { and clad surrounded by moderator. The guide tube } \\
\text { may also contain a burnable poison or control rod } \\
\text { modeled as concentric cylinders (see Figure 2) }\end{array}$ \\
\hline Modeled as two concentric cylinders of moderator \\
and clad surrounded by moderator. The instru- \\
ment tube may also contain a detector modeled as \\
concentric cylinders
\end{tabular}



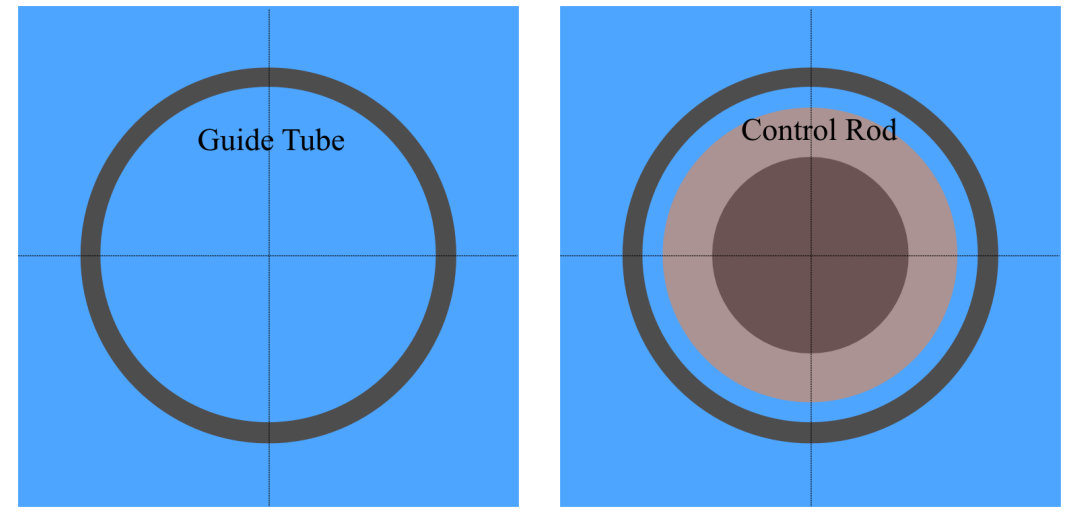

Figure 2: Metadata model examples of guide tube with and without a control rod.

homogenized cross sections for a unit pin cell is described in $\S 3$.

For pin-resolved grids, a separate unit pin cell calculation is carried out to calculate the resonance self-shielded cross sections in each material region of the pin (see §3). These are then weighted with the volume fractions of each region bisecting the mesh cell to calculate an effective volume-weighted set of cross sections as described below. The default behavior is to use pin-resolved meshing for $\mathrm{S}_{\mathrm{N}}$ and pin-homogenized meshing for $\mathrm{SP}_{\mathrm{N}}$; however, each option is available for both transport methods. Details of the meshing process can be found in Appendix A.

After each pin cell is meshed, the complete computational mesh is assembled and partitioned across the available processors. Partitioning is in the $(x, y)$ (radial) dimensions because Denovo uses the KBA wavefront algorithm for $\mathrm{S}_{\mathrm{N}}[1,4,6]$. For compatibility reasons, this decomposition is also used for $\mathrm{SP}_{\mathrm{N}}$. Partitioning attempts to balance the number of computational cells on each domain. Alternatively, an option is available to only allow domain partitioning at physical pin cell boundaries. The two partitioning cases are shown in Fig. 4. Partitioning over computational cells results in better load balancing, especially in full core problems with baffles and reflector regions. However, the domain boundaries can split pin cell boundaries, which means that the same cross section processing will be performed on multiple domains. This cross section processing time is typically small for start-up calculations where the number of unique fuel pins is small. However, for coupled multiphysics problems in which fuel pins at many different temperatures are present, 

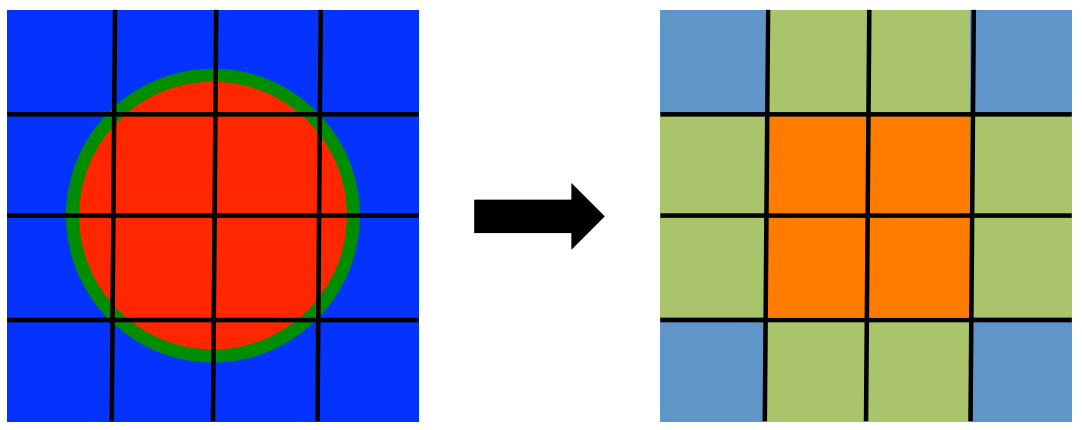

(a) Pin-resolved meshing.
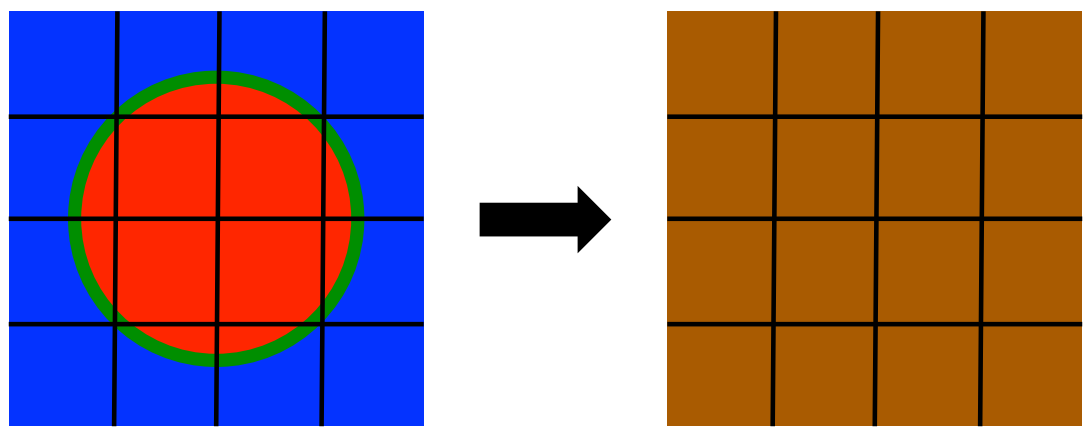

(b) Pin-homogenized meshing.

Figure 3: Sample material homogenization approaches for a single pin cell with a $4 \times 4$ mesh. 
the time spent processing cross sections often dominates the transport time $\left(\right.$ for $\mathrm{SP}_{\mathrm{N}}$ ).

\section{Cross Section Processing}

In this section, we discuss the generation of nuclear data using the XSProc module of the SCALE package [10]. Cross section processing with XSProc is based around local 0-D or 1-D approximations to the reactor geometry. For the PWR geometries targeted by Insilico, this is accomplished using standard Wigner-Seitz approximations [11], converting square lattice cells to concentric circles, allowing a 1-D representation as shown in Fig. 5. The radius of the outermost region is chosen to preserve the moderator volume in the unit cell.

XSProc performs resonance self-shielding calculations using the Bondarenko method [12] with either narrow or intermediate resonance approximations. In addition, an optional 1-D cylindrical discrete ordinates eigenvalue calculation can be used to compute space- and energy-dependent fluxes that are used to perform spatial homogenization and/or energy group collapse. Details about individual modules available within XSProc can be found in Ref. [10].

Insilico supports either "pin-resolved" or "pin-homogenized" cross section generation. In the pin-resolved case, no spatial homogenization is performed in XSProc, and the cross sections for a given mesh cell are computed as a volume-weighted average of the materials located in that mesh cell. A more accurate approach would use radial flux information from the cross section processing calculation to produce flux-weighted cross sections for each mesh cell; however, this information is not currently accessible through XSProc. With pin-homogenized cross sections, XSProc performs flux-weighted spatial homogenization of all of the materials within a given unit cell, and every mesh cell corresponding to that unit cell is assigned the same homogenized data. These two approaches are illustrated in Fig. 3. Because the volume weighting used in the pin-resolved approach does not preserve reaction rates, limited accuracy is achievable without resorting to very fine spatial meshes in the transport calculation. Due to this restriction, only pin homogenization is considered for the results presented in $\S 5$.

Within Insilico several modifications are made to the unit cells provided to XSProc. For unit cells located adjacent to assembly gap regions, the outer radius of the Wigner-Seitz cell is increased to account for the added moderator volume in the gap. For unit cells that do not contain fuel material 


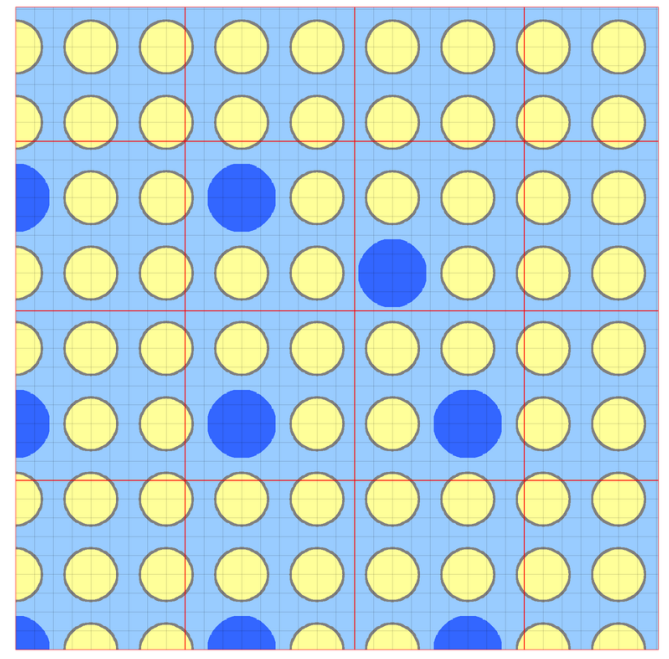

(a) Partitioning balancing computational mesh cells on each domain.

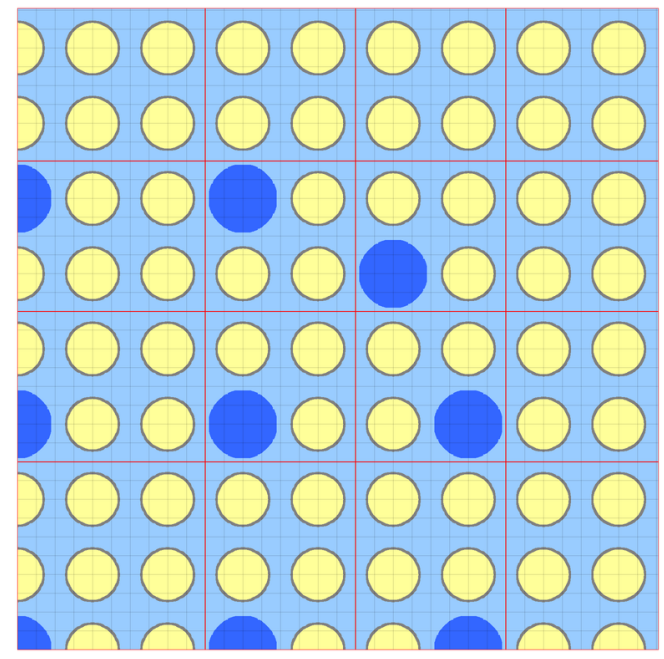

(b) Partitioning constrained by physical pin cell boundaries.

Figure 4: A quarter $17 \times 17$ assembly partioned into a $4 \times 4$ domain. Each pin is meshed with an $8 \times 8$ grid. 

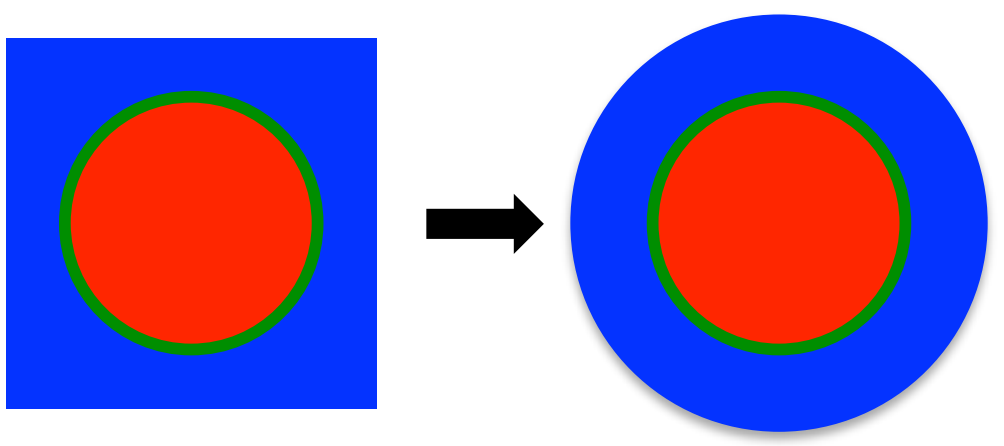

Figure 5: Conversion of unit cell to Wigner-Seitz approximation.

(e.g., guide tubes, control rods), the discrete ordinates eigenvalue calculation is no longer valid. To remedy this, the equivalent cell is modified by adding an extra outer region containing a volume-homogenized mixture of $\mathrm{UO}_{2}$ fuel, zircalloy cladding, and water, with a thickness of one-half of the unit cell pitch. The presence of this fuel buffer allows a standard eigenvalue calculation to be performed, with optional spatial homogenization taking place over only the original materials in the cell. In regions outside the reactor core (e.g., structural materials, outer reflectors), an infinite homogeneous fixed-source calculation is performed rather than an eigenvalue calculation. The source term for this fixed-source calculation is taken to be the fission spectrum of $\mathrm{U}-235$. The final adjustment made by Insilico is in fuel pins containing Westinghouse Integrated Fuel Burnable Absorber (IFBA), a very thin (typically 5-10 $\mu \mathrm{m}$ ) coating of $\mathrm{ZrB}_{2}$ applied to the surface of select fuel pins for reactivity control. The presence of a very thin, strongly absorbing region results in large errors from the XSProc discrete ordinates calculation when using the default settings (an $\mathrm{S}_{8}$ angular quadrature and an automatically generated coarse spatial mesh). To improve the accuracy of XSProc, Insilico adjusts the parameters of the discrete ordinates calculation within cells containing IFBA to use an $\mathrm{S}_{48}$ angular quadrature and a spatial mesh with twice the resolution of the default mesh.

\section{Radiation Transport Models}

As discussed in $\S 1$, Insilico provides an interface to both Monte Carlo (Shift) and deterministic (Denovo) radiation transport solvers. The capabilities of Shift are detailed in Ref. [2]. Denovo supports both discrete ordinates 
$\left(\mathrm{S}_{N}\right)$ and simplified spherical harmonics $\left(\mathrm{SP}_{\mathrm{N}}\right)$ angular discretizations of the transport equation. Although Denovo itself supports the solution of fixedsource transport problems, the interface through Insilico exclusively solves discretized forms of the $k$-eigenvalue Boltzmann neutron transport equation:

$$
\begin{aligned}
\hat{\boldsymbol{\Omega}} \cdot \nabla \psi(\hat{\boldsymbol{\Omega}}, E)+\sigma(E) \psi(\hat{\boldsymbol{\Omega}}, E)= \\
\\
\quad \int_{0}^{\infty} \int_{4 \pi} \sigma_{\mathrm{s}}\left(\hat{\boldsymbol{\Omega}}^{\prime} \rightarrow \hat{\boldsymbol{\Omega}}, E^{\prime} \rightarrow E\right) \psi\left(\hat{\boldsymbol{\Omega}}^{\prime}, E^{\prime}\right) \mathrm{d} \hat{\boldsymbol{\Omega}}^{\prime} \mathrm{d} E^{\prime} \\
+\frac{1}{k} \chi(E) \int_{0}^{\infty} \nu \sigma_{\mathrm{f}}\left(E^{\prime}\right) \int_{4 \pi} \psi\left(\hat{\boldsymbol{\Omega}}^{\prime}, E^{\prime}\right) \mathrm{d} \hat{\boldsymbol{\Omega}}^{\prime} \mathrm{d} E^{\prime} .
\end{aligned}
$$

Here, the state is defined by the angular flux $\psi$ and the independent variables are $\mathbf{r}=(x, y, z)$ in cm, $\hat{\boldsymbol{\Omega}}=(\theta, \varphi)$ in sr, and $E$ in $\mathrm{eV}$, representing space, angle, and energy, respectively. The total, scattering, and fission cross sections are in $\mathrm{cm}^{-1}$ and are given by $\sigma, \sigma_{\mathrm{s}}$, and $\sigma_{\mathrm{f}}$, respectively, and the fission spectrum is $\chi$. The angular flux and all material properties are a function of $\mathbf{r}$, which has been suppressed for brevity. $k$ is an eigenvalue of the system. The dominant eigenvalue represents the multiplication factor of the system such that $k=1$ represents a reactor in sustained (critical) steady-state operation. Both the $\mathrm{S}_{\mathrm{N}}$ and $\mathrm{SP}_{\mathrm{N}}$ solvers are based around the multigroup energy discretization in which the continuum of particle energies is divided into discrete bins, with material properties and solution variables appropriately averaged over individual bins [13].

The $S_{N}$ angular discretization is a collocation method in which Eq. (1) is enforced only for a finite set of discrete angles; integrals over angle are then evaluated using quadrature rules over the unit sphere. A full derivation of the discrete ordinates equations can be found in Ref. [1]. The discrete angles and corresponding quadrature weights, commonly referred to as a quadrature set, are selected to allow certain symmetry and accuracy conditions (e.g., exactly integrating the maximum number of spherical harmonic functions). An investigation into the selection of quadrature sets for reactor eigenvalue problems can be found in Ref. [14].

Denovo offers a wide variety of spatial discretizations for the discrete ordinates equations, including step characteristics and linear discontinuous finite elements. A detailed description of the equation types can be found in Ref. [1]. Denovo offers both power iteration and Arnoldi eigensolvers as well as both source iteration and Krylov subspace linear solvers. Krylov subspace solvers use the Trilinos library [15]. 
The $\mathrm{SP}_{\mathrm{N}}$ angular approximation was developed as a straightforward extension of the 1-D $\mathrm{P}_{\mathrm{N}}$ equations to higher dimensions. By eliminating the odd moment orders from the set of equations and applying a linear transformation, the $\mathrm{SP}_{\mathrm{N}}$ equations can be reduced to a series of diffusion-like equations coupled in both energy and angular moment. The $\mathrm{SP}_{\mathrm{N}}$ equations can be written as

$$
-\nabla \cdot \mathbb{D}_{n} \nabla \mathbb{U}_{n}+\sum_{m=1}^{4} \mathbb{A}_{n m} \mathbb{U}_{m}=\frac{1}{k} \sum_{m=1}^{4} \mathbb{F}_{n m} \mathbb{U}_{m}, \quad n=1,2, \ldots, \frac{N+1}{2},
$$

where $\mathbb{U}_{n}$ is the vector of transformed angular moments, the matrices $\mathbb{D}_{n}$ contain the multigroup diffusion coefficients, $\mathbb{A}_{n}$ contains the total cross section and scattering data, and $\mathbb{F}_{n m}$ contains the fission terms. Unlike standard multigroup diffusion approximations, which use simplifications to decouple the diffusion coefficients in energy in order to produce a series of monoenergetic equations, the diffusion coefficients in Eq. (2) are fully coupled in energy. Because of this, the $\mathrm{SP}_{1}$ equation described here is completely consistent with the $\mathrm{P}_{1}$ equations, whereas a standard multigroup diffusion formulation is not [11]. The Denovo $\mathrm{SP}_{\mathrm{N}}$ implementation does additionally support outscattercorrected diffusion coefficients, in which a standard multigroup diffusion equation is recovered with diffusion coefficients that are decoupled in energy. This approximation results in a slightly sparser matrix formulation with a corresponding reduction in memory requirements.

The Denovo $\mathrm{SP}_{\mathrm{N}}$ solver supports both reflecting and vacuum boundaries. Vacuum boundaries are implemented using a Marshak condition, which results in coupling between energy groups and angular moments on the problem boundary. Numerous eigenvalue solvers are available, including power iteration, Arnoldi's method, Rayleigh quotient iteration, and a generalized Davidson solver. Krylov subspace linear solvers, along with several algebraic preconditioning options, are also available. Further details about the derivation of the $\mathrm{SP}_{\mathrm{N}}$ equations and a comparison of eigenvalue solvers can be found in Ref. [16].

It was shown in Ref. [17] that due to the volume-weighting of cross sections described in $\S 3$, a very fine mesh resolution (on the order of a $12 \times 12$ radial mesh per pin cell) was necessary to produce accurate estimates of core-wide eigenvalues. Subsequent efforts have shown that in order to produce accurate power distributions, even finer mesh resolutions are necessary, especially in the presence of strongly absorbing materials. Although it has been shown 
that the Denovo $\mathrm{S}_{\mathrm{N}}$ solver has excellent parallel scaling properties [6], the computational resources necessary to produce accurate results for full reactor calculations are substantial and far beyond those available to most reactor analysts. For this reason, the numerical results presented in $\S 5$ are limited to the Denovo $\mathrm{SP}_{\mathrm{N}}$ discretization.

\section{Computational Results}

To evaluate the accuracy of the pin-homogenized $\mathrm{SP}_{\mathrm{N}}$ implementation in Insilico, we consider three problems. The first two test cases are problems 2 and 5 from the CASL VERA core physics benchmark progression problems [18]. The final test case is a full core model of the Westinghouse AP $1000^{\circledR}$ reactor design. In all cases, 23-group cross sections are collapsed from a 252 group library as described in $\S 3$. An $\mathrm{SP}_{5}$ angular order is used in conjunction with $\mathrm{P}_{3}$ anisotropic scattering; the coefficients of the $\mathrm{SP}_{\mathrm{N}}$ equations are computed based on the homogenized multigroup cross sections using the full $\mathrm{P}_{\mathrm{N}}$ scattering matrices. Each pin cell uses a $2 \times 2$ spatial discretization in the $x-y$ plane.

\subsection{VERA Problem 2}

VERA Problem 2 is a two-dimensional array of fuel rods representative of a typical PWR fuel assembly. The rods are located in a $17 \times 17$ square pitch lattice pattern with 264 fuel pins, 24 guide tube locations, and a single central instrumentation tube based on Westinghouse assembly specifications. The fuel consists of $3.1 \%$ enriched $\mathrm{UO}_{2}$. Seventeen different configurations are considered, consisting of various different fuel temperatures, guide tube inserts, and burnable absorbers. Cases L, M, and N contain IFBA burnable absorbers, and those pins therefore use a higher-resolution transport calculation to perform spatial homogenization and energy group collapse, as described in $\S 3$. Full geometric and material descriptions can be found in Ref. [18]. The benchmark results were computed using continuous-energy Monte Carlo using the KENO-VI code in the SCALE package. The uncertainty in the eigenvalue for all cases was \pm 3 per cent mille (pcm).

Table 2 provides the reference eigenvalue for each configuration as well as the error in the eigenvalue and fission rate distributions computed by Insilico using the $\mathrm{SP}_{\mathrm{N}}$ solver. Both the computed eigenvalues and fission rate distributions are in excellent agreement with the reference solution, with the eigenvalue difference exceeding 200 pcm in only two cases. These results 
indicates that the $\mathrm{SP}_{\mathrm{N}}$ solver is able to compute both eigenvalues and spatial power distributions with a high level of accuracy, even in the presence of strongly absorbing media. Only in the configuration containing strongly absorbing $\mathrm{B}_{4} \mathrm{C}$ control rods did the root mean square (RMS) fission rate error exceed $0.5 \%$ and the maximum fission rate error exceed $1 \%$. There is a mild negative bias with increasing temperature observable in cases A through D. A similar bias of nearly the same magnitude was seen using the MPACT lattice physics solver in Ref. [19], using the same underlying data. In Ref. [20], this temperature bias was attributed to the lack of temperature-dependent scattering data in the cross section libraries used by MPACT (and also by Insilico). That study offered a modified approach to the generation of cross section libraries that significantly improved the accuracy of MPACT at higher temperatures. This approach has not yet been implemented in XSProc.

Insilico can also run the $\mathrm{S}_{\mathrm{N}}$ solver from the same problem inputs. Figure 6 shows the error in $k_{\text {eff }}$ for $\mathrm{S}_{\mathrm{N}}$ results as compared to the KENO-VI benchmarks. Each calculation was done using a $20 \times 20$ mesh per pincell and volumeweighted cross sections. The angular quadrature used a quadruple range set with 2 polar and 8 azimuthal angles per quadrant [14]. The $\mathrm{S}_{\mathrm{N}}$ results are consistently below the benchmark and are characterized by large $(\sim 1000 \mathrm{pcm})$ errors in problems with small scale features such as IFBA.

To test the convergence properties of the $\mathrm{S}_{\mathrm{N}}$ solver, we performed a mesh refinement study for both 23 and 56 group cross section libraries. These results are shown in Fig. 7 . The 23 group library consistently undershoots the benchmark result. The 56 group library converges towards the benchmark at a $20 \times 20$ pincell mesh. However, as the mesh is further refined, the solution

overshoots the benchmark. These results show the difficulty of applying regular-grid $\mathrm{S}_{\mathrm{N}}$ to reactor problems. The space-angle-energy discretization must be refined consistently. In other words, refining in space without refining in energy or angle is insufficient.

\subsection{VERA Problem 5}

The second benchmark problem is VERA Problem 5, a quarter-core model of the initial criticality and zero power physics testing at the Watts Bar Nuclear Power Station, Unit 1 (WBN1). Because the measurements are performed at zero power, no simulation of thermal-hydraulics behavior is required to model the reactor conditions, and because the initial start-up contains only fresh fuel, no depletion modeling is necessary. Therefore, this benchmark represents a rare opportunity to compare numerical simulations to measured data on the 


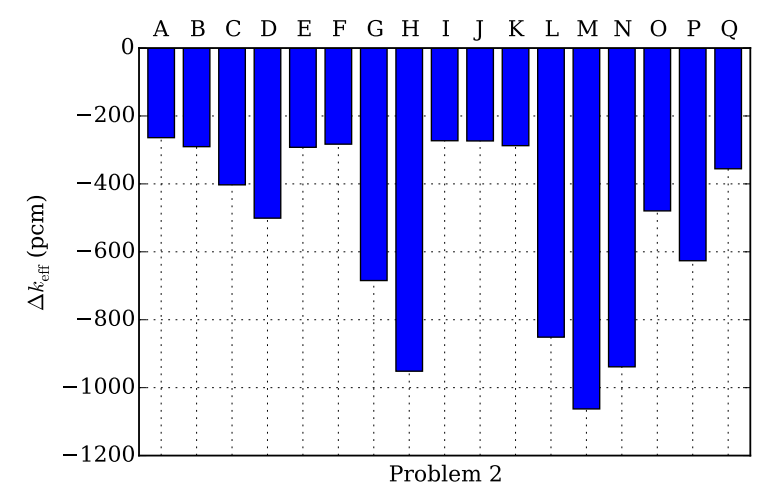

Figure 6: The difference between $k_{\text {eff }}$ computed by Insilico using $\mathrm{S}_{\mathrm{N}}$ solver and KENO-VI benchmark results for VERA Problem 2.

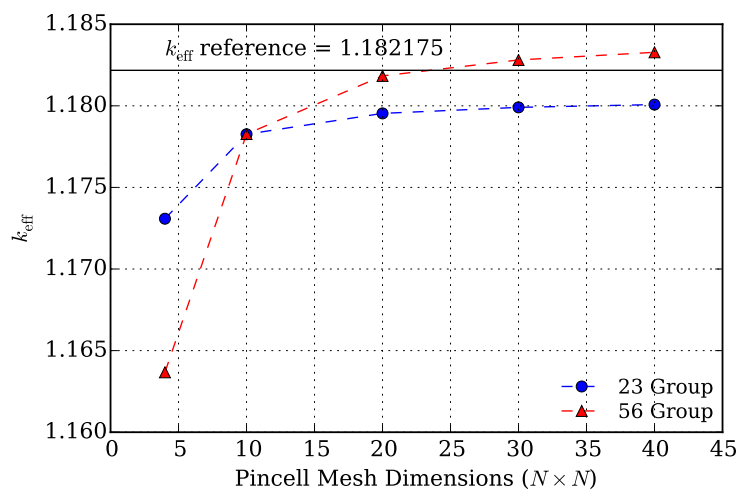

Figure 7: Variation of eigenvalue as a function of mesh size for 23 and 56 group libraries using Insilico with $\mathrm{SP}_{\mathrm{N}}$ solver. 
Table 2: Insilico eigenvalue and fission rate distribution comparisons for VERA Problem 2 using $\mathrm{SP}_{\mathrm{N}}$ solver.

\begin{tabular}{cccccc}
\hline \multirow{2}{*}{ Case } & Description & $\begin{array}{c}\text { KENO-VI } \\
\text { eff }\end{array}$ & $\begin{array}{c}\Delta k_{\text {eff }} \\
{[ \pm \mathbf{3} \mathbf{~ p c m}]}\end{array}$ & \multicolumn{2}{c}{ Fission Rate Error } \\
\cline { 5 - 6 } & & 1.182175 & -19 & 0.19 & 0.39 \\
$\mathbf{A}$ & $565 \mathrm{~K}$ & 1.183360 & -43 & 0.15 & 0.34 \\
$\mathbf{B}$ & $600 \mathrm{~K}$ & 1.173751 & -122 & 0.15 & 0.32 \\
$\mathbf{C}$ & $900 \mathrm{~K}$ & 1.165591 & -221 & 0.14 & 0.24 \\
$\mathbf{D}$ & $1200 \mathrm{~K}$ & 1.069627 & 96 & 0.11 & 0.26 \\
$\mathbf{E}$ & 12 Pyrex & 0.976018 & 170 & 0.18 & 0.40 \\
$\mathbf{F}$ & 24 Pyrex & 0.847695 & -142 & 0.33 & 0.65 \\
$\mathbf{G}$ & 24 AIC & 0.788221 & -547 & 0.59 & 1.27 \\
$\mathbf{H}$ & 24 B 4 C & 1.179916 & -18 & 0.16 & 0.34 \\
$\mathbf{I}$ & Instrument Thimble & 0.975193 & 180 & 0.18 & 0.38 \\
$\mathbf{J}$ & Instr. + 24 Pyrex & 1.020063 & 172 & 0.17 & 0.39 \\
$\mathbf{K}$ & Zoned + 24 Pyrex & 1.018915 & -81 & 0.37 & 0.70 \\
$\mathbf{L}$ & 80 IFBA & 0.938796 & -108 & 0.41 & 0.59 \\
$\mathbf{M}$ & 128 IFBA & 0.869615 & 17 & 0.28 & 0.60 \\
$\mathbf{N}$ & 104 IFBA + 20 WABA & 1.047729 & -37 & 0.28 & 0.68 \\
$\mathbf{O}$ & 12 Gadolinia & 0.927410 & -24 & 0.32 & 0.87 \\
$\mathbf{P}$ & 24 Gadolinia & 1.171940 & -147 & 0.20 & 0.56 \\
$\mathbf{Q}$ & Zircaloy Spacer Grid & Average & $\mathbf{5 1}$ & $\mathbf{0 . 2 5}$ & $\mathbf{0 . 5 3}$ \\
\hline
\end{tabular}


level of an entire reactor core. The WBN1 reactor is a four-loop PWR of typical Westinghouse design, containing $19317 \times 17$ fuel assemblies. Three different fuel enrichments, eight control rod banks, and numerous Pyrex burnable poison rods are located throughout the core. Detailed geometric, material, and operating specifications can be found in Ref. [21]. As described in that specification, all materials are at a constant temperature of $565 \mathrm{~K}$. The total number of unknowns in the Insilico problem is approximately $1.2 \times 10^{9}$. The Insilico results were each executed using 676 compute cores on OLCF's Titan computer [22]. The initial criticality calculation took 33 minutes to complete, including both cross section processing and the eigenvalue calculation; run times for the remaining cases were similar. By comparison, each KENO-VI calculation required approximately 44 hours on 300 compute cores.

Table 3 shows the eigenvalues and deviations from measured data for both KENO-VI and Insilico for ten different critical configurations visited during the calculation of control rod bank worths. The results show an excellent agreement between both numerical approaches and the measured data, with the maximum deviation over all cases being less than $150 \mathrm{pcm}$. Even more remarkable is the nearly perfect agreement between the KENO-VI continuous energy results and those produced by Insilico with the $\mathrm{SP}_{\mathrm{N}}$ solver, with deviations of less than $25 \mathrm{pcm}$ in all cases. Table 4 shows the measured and computed values for the differential boron worth. Although there is a slight discrepancy between the measured value and those computed numerically, there is excellent agreement between the two codes. No comparison of localized quantities (such as pin fission rates) was possible because the reference Monte Carlo solutions were unable to compute such quantities with a sufficiently small uncertainty to allow a meaningful comparison.

\subsection{Westinghouse AP1000 ${ }^{\circledR}$}

The final problem selected is an analysis of the Westinghouse AP $100{ }^{\circledR}$ reactor core. Although similar geometrically to WBN1, the AP1000 ${ }^{\circledR}$ contains a number of features that present a more significant challenge for core analysis methods. In particular, the AP $1000^{\circledR}$ core uses a wider range of fuel enrichments, several different burnable absorbers such as IFBA and Wet Annular Burnable Absorber (WABA), and multiple control rod materials. Detailed specifications for the AP1000 ${ }^{\circledR}$ can be found in Ref. [23]. As with

VERA Problem 5, the problem is modeled at a constant temperature of $565 \mathrm{~K}$. Each Insilico case required approximately 100 minutes of computational time 
Table 3: VERA Problem 5 Control Rod Bank Worth

\begin{tabular}{|c|c|c|c|c|c|c|}
\hline \multirow[t]{2}{*}{$\begin{array}{c}\text { Case } \\
\text { Description }\end{array}$} & \multirow{2}{*}{$\begin{array}{c}\text { Critical } \\
\text { Boron } \\
{[\mathrm{ppm}]}\end{array}$} & \multirow{2}{*}{$\begin{array}{c}\text { Ref. Bank } \\
\text { Position } \\
\text { [steps] }\end{array}$} & \multicolumn{2}{|c|}{$k_{\text {eff }}$} & \multicolumn{2}{|c|}{$\begin{array}{c}\text { Reactivity } \\
\text { Difference }[\mathrm{pcmB}]\end{array}$} \\
\hline & & & KENO-VI & Insilico & KENO-VI & Insilico \\
\hline Initial & 1293 & 167 & 0.99957 & 0.99981 & -43 & -19 \\
\hline $\mathrm{ARO}^{\dagger}$ & 1299 & 230 & 1.00016 & 1.00024 & 16 & 24 \\
\hline Bank D In & 1177 & 18 & 0.99894 & 0.99884 & -106 & -116 \\
\hline Bank C In & 1177 & 119 & 0.99886 & 0.99884 & -114 & -116 \\
\hline Bank B In & 1177 & 113 & 0.99906 & 0.99927 & -94 & -73 \\
\hline Bank A In & 1177 & 97 & 0.99863 & 0.99853 & -138 & -147 \\
\hline Bank SD In & 1177 & 71 & 0.99878 & 0.99878 & -122 & -122 \\
\hline Bank SC In & 1177 & 71 & 0.99873 & 0.99876 & -127 & -124 \\
\hline Bank SB In & 1177 & 134 & 0.99911 & 0.99921 & -89 & -79 \\
\hline \multirow[t]{3}{*}{ Bank SA In } & 1177 & 69 & 0.99872 & 0.99883 & -128 & -117 \\
\hline & & Average & 0.99906 & 0.99911 & -94 & -89 \\
\hline & Std & Deviation & 0.00047 & 0.00054 & 48 & 54 \\
\hline
\end{tabular}

${ }^{\dagger}$ all rods out

Table 4: VERA Problem 5 Differential Boron Worth

\begin{tabular}{ccc}
\hline Method & $\begin{array}{c}\text { DBW } \\
{[\mathbf{p c m} / \mathbf{p p m B}]}\end{array}$ & $\begin{array}{c}\text { Difference } \\
{[\mathbf{p c m} / \mathbf{p p m B}]}\end{array}$ \\
\hline Measured & -10.77 & - \\
KENO-VI & -10.21 & 0.56 \\
Insilico & -10.16 & 0.61 \\
\hline
\end{tabular}


Table 5: VERA AP1000 ${ }^{\circledR}$ HZP Reactivity Results

\begin{tabular}{ccccc}
\hline Case & Units & KENO-VI & Insilico & Difference \\
\hline$k_{\text {eff at 1321 ppm }}$ & - & 1.00066 & 1.00033 & -33 \\
Critical Boron & $\mathrm{ppm}$ & 1313 & 1310 & -3 \\
Boron Worth & $\mathrm{pcm} / \mathrm{ppm}$ & -9.6 & -9.4 & +0.2 \\
\hline
\end{tabular}

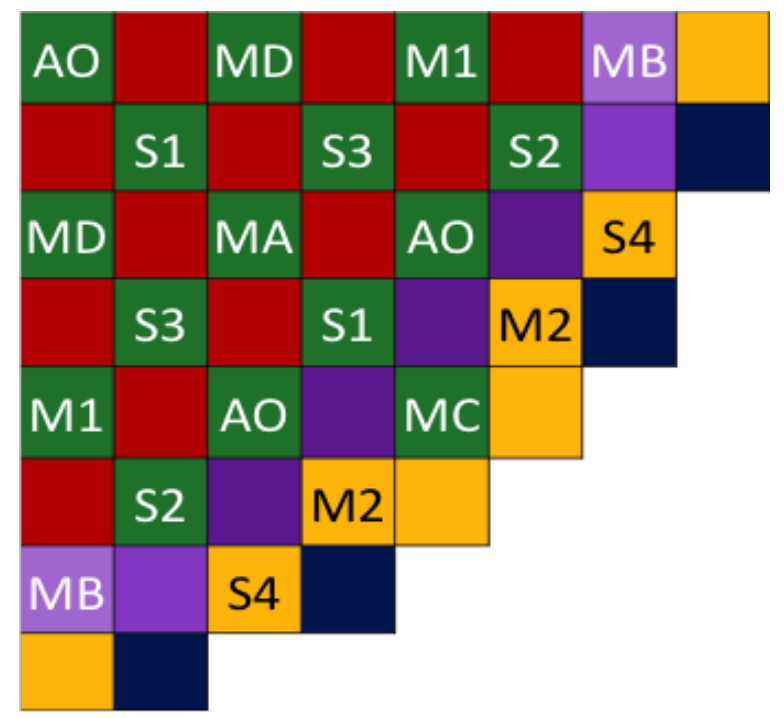

Figure 8: AP1000 ${ }^{\circledR}$ Control Rod Bank Locations.

on 320 compute cores, while the KENO-VI reference solutions needed 6.5 days on 180 cores.

Because no reactors of this design have yet become operational, this benchmark is performed as a code-to-code comparison, with KENO-VI again providing the reference solution. Table 5 provides a comparison of several reactivity-related quantities and Table 6 shows the control rod bank worths (the locations of which are shown in Fig. 8) computed by KENO-VI and Insilico as well as the corresponding deviations between the codes. Once again, the agreement between KENO-VI and Insilico is striking, with all cases agreeing within $10 \mathrm{pcm}$. The difficulty in achieving a suitably small uncertainty in localized quantities again prevented code-to-code comparison of more detailed information such as pin fission rates. 
Table 6: VERA AP1000 ${ }^{\circledR}$ Control Rod Bank Worth

\begin{tabular}{cccccc}
\hline \multirow{2}{*}{ Bank } & \multirow{2}{*}{ Material } & \multicolumn{2}{c}{ Bank Worth } & $\Delta$ Worth & $\Delta$ Worth \\
\cline { 3 - 4 } & & KENO-VI & Insilico & {$[\mathrm{pcm}]$} & {$[\%]$} \\
\hline MA & Tungsten & 258 & 257 & -1 & -0.5 \\
MB & Tungsten & 217 & 212 & -5 & -2.1 \\
MC & Tungsten & 188 & 186 & -2 & -1.1 \\
MD & Tungsten & 234 & 234 & 0 & 0.0 \\
M1 & Ag-In-Cd & 651 & 647 & -4 & -0.6 \\
M2 & Ag-In-Cd & 887 & 890 & 3 & 0.4 \\
AO & Ag-In-Cd & 1635 & 1631 & -4 & -0.3 \\
S1 & Ag-In-Cd & 1079 & 1079 & 0 & 0.0 \\
S2 & Ag-In-Cd & 1096 & 1087 & -9 & -0.8 \\
S3 & Ag-In-Cd & 1124 & 1124 & 0 & 0.0 \\
S4 & Ag-In-Cd & 580 & 577 & -3 & -0.4 \\
\hline
\end{tabular}

\section{Conclusions}

Insilico is a new tool for computational modeling of light water reactors with initial emphasis on PWR designs. Insilico offers a simple, unified interface to both Monte Carlo and deterministic radiation transport solvers. The Monte Carlo capabilities are described in a companion paper [2] while the deterministic discretizations, consisting of both $\mathrm{S}_{\mathrm{N}}$ and $\mathrm{SP}_{\mathrm{N}}$ approximations, are described in this work. Automated cross section processing of local unit cell configurations is provided using the XSProc module of SCALE; both spatial homogenization and energy group collapse from standard AMPX cross section libraries are available.

Numerical results for several PWR test problems, including multiple problems from the set of CASL VERA core physics benchmark suite as well as a detailed model of the Westinghouse AP1000 ${ }^{\circledR}$ reactor design were produced. These problems exhibit a wide range of features that typically present difficulties for reactor analysis tools, including control rods and various burnable absorbers (e.g., IFBA, WABA, Pyrex). These numerical results indicate that the $\mathrm{SP}_{\mathrm{N}}$ discretization with pin-homogenized cross sections is 
capable of delivering very accurate solutions, with favorable comparisons against both continuous-energy Monte Carlo solutions and measured plant operating data. The accuracy of the solution process is displayed not only with accurate calculation of pin powers, but also with control rod worth and differential boron worth. For the Watts Bar core, the average deviation from plant measured control rod worths is less than $100 \mathrm{pcm}$, and the average deviation from values computed by continuous-energy Monte Carlo is less than $10 \mathrm{pcm}$. For the more advanced AP $1000{ }^{\circledR}$ core, the average deviation in control rod worth as compared to Monte Carlo was less than $5 \mathrm{pcm}$, or around $0.6 \%$. Because there are no $\mathrm{AP} 1000^{\circledR}$ reactors yet operational, comparison to measured data is not yet possible.

These numerical results give a strong indication that Insilico can serve as a valuable tool for design and analysis of PWR designs. Although the $\mathrm{SP}_{\mathrm{N}}$ approximation will ultimately be unable to deliver the same accuracy as Monte Carlo calculations, the ability to run full core reactor analyses on only a few hundred processors potentially makes this approach a viable option for many more reactor analysts.

\section{Acknowledgements}

Work for this paper was supported by Oak Ridge National Laboratory, which is managed and operated by UT-Battelle, LLC, for the U.S. Department of Energy under Contract No. DEAC05-00OR22725. This research was supported by the Consortium for Advanced Simulation of Light Water Reactors (www.casl.gov), an Energy Innovation Hub (http://www.energy.gov/hubs) for Modeling and Simulation of Nuclear Reactors under U.S. Department of Energy Contract No. DE-AC05-00OR22725. This research used resources of the Oak Ridge Leadership Computing Facility at the Oak Ridge National Laboratory, which is supported by the Office of Science of the U.S. Department of Energy under Contract No. DE-AC05-00OR22725.

\section{Appendix A. Meshing Details}

A necessary condition for $k$-eigenvalue problems is the exact (to numerical precision) conservation of fissionable material in the problem. Therefore, in pin-resolved grids the volume fractions must preserve the exact fuel volume. Consider the PWR pin cell illustrated in Fig. A.9. Assume, as shown in the figure, that we wish to radially mesh the pin using an $8 \times 8$ grid. The meshing 


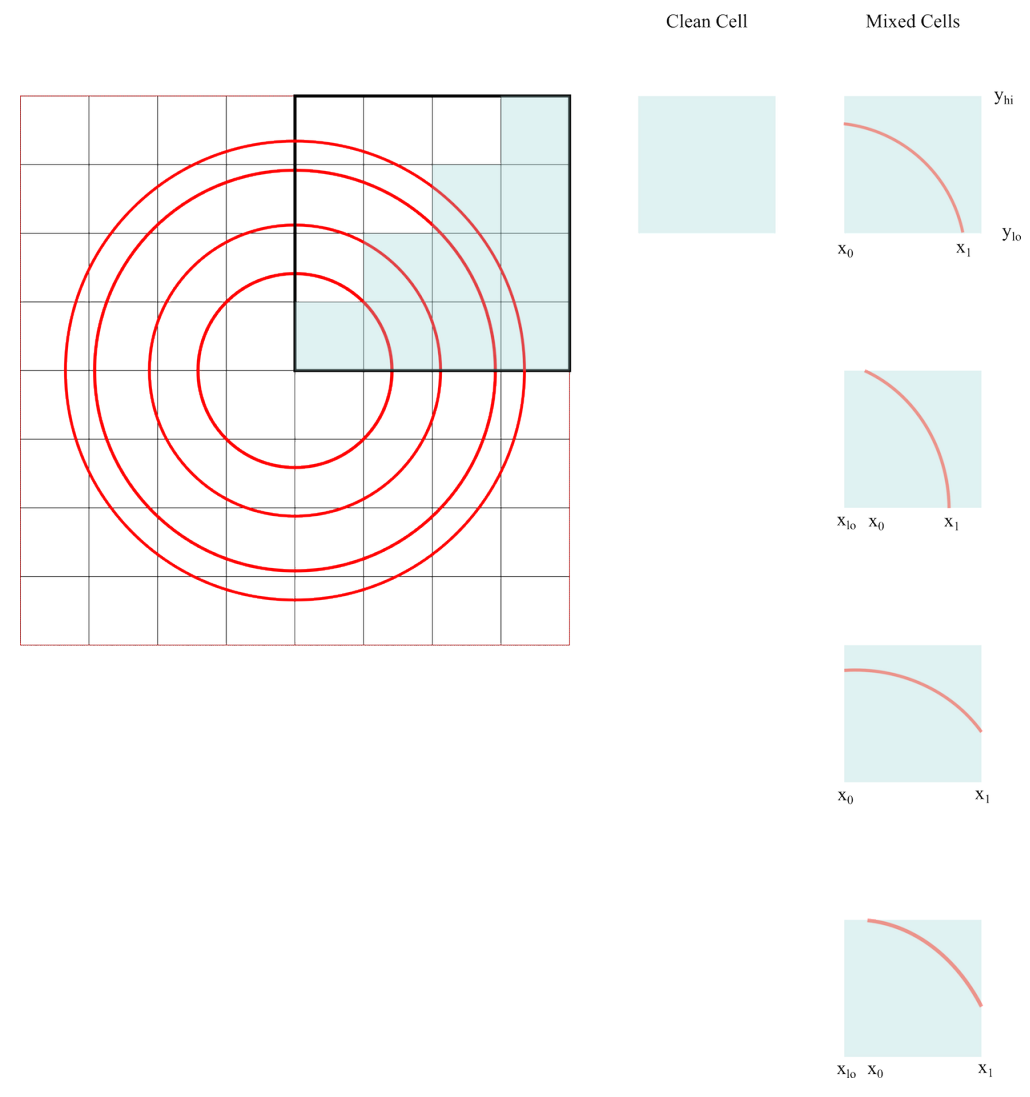

Figure A.9: PWR pin cell meshed with an $8 \times 8$ cartesian grid. There are four types of mixed cells (illustrated on the right). 
Table A.7: Different mixtures produced from meshing the pin cell in Fig. A.9

\begin{tabular}{|l|l|l|l|l|l|l|l|}
\hline 1 & 1 & 7 & 4 & 4 & 7 & 1 & 1 \\
\hline 1 & 8 & 6 & 3 & 3 & 6 & 8 & 1 \\
\hline 7 & 6 & 5 & 2 & 2 & 5 & 6 & 7 \\
\hline 4 & 3 & 2 & 0 & 0 & 2 & 3 & 4 \\
\hline 4 & 3 & 2 & 0 & 0 & 2 & 3 & 4 \\
\hline 7 & 6 & 5 & 2 & 2 & 5 & 6 & 7 \\
\hline 1 & 8 & 6 & 3 & 3 & 6 & 8 & 1 \\
\hline 1 & 1 & 7 & 4 & 4 & 7 & 1 & 1 \\
\hline
\end{tabular}

algorithm uses symmetry to only mesh one-eighth (for $N \times N$ meshes) or one-fourth (for $N \times M$ meshes) of the cells. In this example, the resulting meshing produces nine unique mixtures, as shown in Table A.7. Mixtures 0 and 1 are clean fuel and moderator, respectively. The additional cells are all mixtures of multiple materials. For example, mixture 2 has volume contributions from the first three regions of the pin cell. Insilico treats all objects in the core, including guide tubes, nozzles, pin caps, and other features as pin cells, and each is meshed using the same technique.

The volume subtended by a shell in a cylindrical shell segment can be calculated by integrating

$$
f(x)=\sqrt{r^{2}-x^{2}}
$$

Additionally, we must add any component of the volume in the cell outside the shell segment, and we must subtract the component outside the cell. Thus, we have

$$
V_{\text {shell }}=\int_{x_{0}}^{x_{1}} f(x) d x+\left(y_{\mathrm{hi}}-y_{\mathrm{lo}}\right)\left(x_{0}-x_{\mathrm{lo}}\right)-y_{\mathrm{lo}}\left(x_{1}-x_{0}\right)
$$

where the integral is

$$
\left.\frac{1}{2}\left[x \sqrt{r^{2}-x^{2}}+r^{2} \tan ^{-1}\left(\frac{x}{\sqrt{r^{2}-x^{2}}}\right)\right]\right|_{x_{0}} ^{x_{1}} .
$$

As illustrated in Fig. A.9, $x_{\mathrm{lo}}, x_{\mathrm{hi}}, y_{\mathrm{lo}}$, and $y_{\mathrm{hi}}$ are the low and high boundaries of the mesh cell in each direction. There are four basic types of shell-cell intersections, each of which is illustrated in the figure. This algorithm is only 

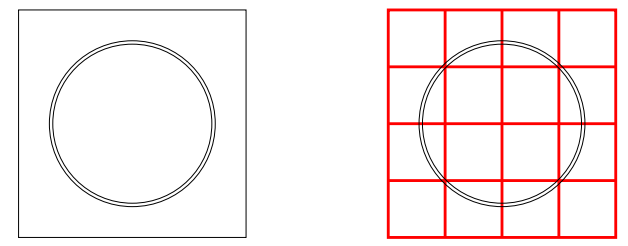

\begin{tabular}{|l|l|l|l|}
\hline 2 & 1 & 1 & 2 \\
\hline 1 & 0 & 0 & 1 \\
\hline 1 & 0 & 0 & 1 \\
\hline 2 & 1 & 1 & 2 \\
\hline
\end{tabular}
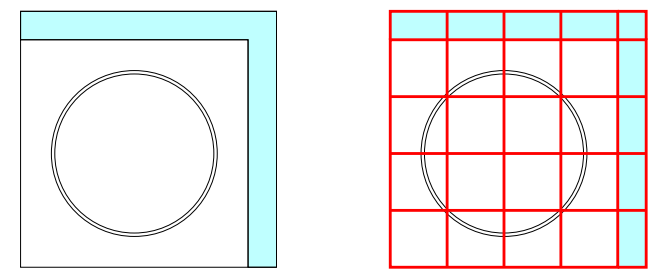

\begin{tabular}{|l|l|l|l|l|}
\hline 3 & 4 & 4 & 5 & 5 \\
\hline 3 & 4 & 4 & 5 & 5 \\
\hline 1 & 0 & 0 & 4 & 4 \\
\hline 1 & 0 & 0 & 4 & 4 \\
\hline 2 & 1 & 1 & 3 & 3 \\
\hline
\end{tabular}

Figure A.10: Visualization of computational mesh overlaying a regular pin cell and an assembly corner pin cell with an adjacent assembly gap.

valid for $N \times N$ and $N \times M$ meshes in which $N$ and $M$ are positive, even integers. The final volumes for each region in the pin cell are obtained by iterating over the shells and subtracting the inner shell volume from the outer shell volume. The outermost region (moderator) of the pin cell has volume $V_{c}-V_{\text {outer }}$ where $V_{c}$ is the volume of the mesh cell and $V_{\text {outer }}$ is the volume of the outermost shell.

Pin cells that are adjacent to assembly boundaries can have a small water gap that is generally not more than $0.5 \mathrm{~cm}$ thick. By default, this gap is meshed with a single computational cell, as illustrated in Fig. A.10. In order to properly couple with thermohydraulics codes, the gap region must have the same temperature as the adjacent pin cell moderator. To achieve this, the gap cell is given the same mixture as the adjacent computational cell in the pin. Thus, when volume fractions are being calculated, the volume of the gap region must be added to both the moderator volume and the total volume of the computational mesh cell.

Once the volumes in each mesh cell are known, the cross section for mesh cell $c$ can be constructed from the macroscopic cross sections in each region $r$ via

$$
\sigma_{c}=\sum_{r}^{N_{r}} \sigma_{r} f_{r}, \quad c=0,1,2, \ldots N_{c}-1,
$$

where $N_{c}$ is the number of mesh cells discretizing a pin cell (16 in the example 
shown in Fig. A.9), $N_{r}$ is the number of unique material regions in the pin cell (5 in the example), and $f_{r}=V_{r} / V_{c}$ is the volume fraction for region $r$.

\section{References}

[1] T. Evans, A. Stafford, R. Slaybaugh, K. Clarno, DENOVO: A new three-dimensional parallel discrete ordinates code in SCALE, Nuclear Technology 171 (2010) 171-200.

[2] T. M. Pandya, S. R. Johnson, T. M. Evans, G. G. Davidson, S. P. Hamilton, A. T. Godfrey, Capabilities, implementation, and benchmarking of shift, a massively parallel monte carlo radiation transport code, Journal of Computational Physics submitted.

[3] SCALE: A comprehensive modeling and simulation suite for nuclear safety analysis and design, Tech. Rep. ORNL/TM-2005/39, Version 6.1, Oak Ridge National Laboratory, Oak Ridge, TN (2011).

[4] R. Baker, K. Koch, An $S_{N}$ Algorithm for the Massively Parallel CM-200 Computer, Nuclear Science and Engineering 128 (1998) 312-320.

[5] M. P. Adams, et al., Provably optimal parallel transport sweeps on regular grids, in: International Conference on Mathematics and Computational Methods Applied to Nuclear Science \& Engineering (M\&C2013), Sun Valley, ID, 2013.

[6] G. G. Davidson, T. M. Evans, J. J. Jarrell, S. P. Hamilton, T. M. Pandya, R. N. Slaybaugh, Massively parallel, three-dimensional transport solutions for the $k$-eigenvalue problem, Nuclear Science and Engineering 177 (2) (2014) 111-125.

[7] S. Hamilton, K. Clarno, Mathematical framework for the coupling of the AMP and Denovo codes, Transactions of the American Nuclear Society 105 (2011) 515-517.

[8] S. Hamilton, et al., Multiphysics methods for LWR analysis, in: International Conference on Mathematics and Computational Methods Applied to Nuclear Science \& Engineering (M\&C2013), Sun Valley, ID, 2013. 
[9] M. Ellis, B. Forget, K. Smith, Preliminary coupling of the monte carlo code openmc and the multiphysics object-oriented simulation environment (moose) for analyzing doppler feedback in monte carlo simulations, in: Joint International Conference on Mathematics and Computation (M\&C), Supercomputing in Nuclear Applications (SNA) and the Monte Carlo (MC) Method, Nashville, TN, 2015.

[10] B. Rearden, R. Lefebvre, J. Lefebvre, K. Clarno, M. Williams, L. Petrie, U. Mertyurek, Modernization enhancements in SCALE 6.2, in: PHYSOR 2014 - The Role of Reactor Physics Toward a Sustainable Future, Kyoto, Japan, 2014.

[11] W. M. Stacey, Nuclear Reactor Physics, John Wiley \& Sons, 2001.

[12] J. J. Duderstadt, L. J. Hamilton, Nuclear Reactor Analysis, John Wiley \& Sons, 1976.

[13] E. Lewis, W. Miller, Computational Methods of Neutron Transport, John Wiley and Sons, New York, 1984.

[14] J. J. Jarrell, T. M. Evans, G. G. Davidson, Discrete ordinate quadrature selection for reactor-based eigenvalue problems, in: International Conference on Mathematics and Computational Methods Applied to Nuclear Science \& Engineering (M\&C2013), Sun Valley, ID, 2013.

[15] M. A. Heroux, R. A. Bartlett, V. E. Howle, R. J. Hoekstra, J. J. Hu, T. G. Kolda, R. B. Lehoucq, K. R. Long, R. P. Pawlowski, E. T. Phipps, A. G. Salinger, H. K. Thornquist, R. S. Tuminaro, J. M. Willenbring, A. Williams, K. S. Stanley, An overview of the Trilinos project, ACM Trans. Math. Softw. 31 (3) (2005) 397-423. doi:http://doi.acm.org/10.1145/1089014.1089021.

[16] S. P. Hamilton, T. M. Evans, Efficient solution of the simplified $\mathrm{P}_{N}$ equations, Journal of Computational Physics 285 (2015) 155-170.

[17] J. Jarrell, T. Evans, G. Davidson, A. Godfrey, Full core reactor analysis: Running Denovo on Jaguar, Nuclear Science and Engineering 175 (3) (2013) 283-291. 
[18] A. T. Godfrey, VERA core physics benchmark progression problem specifications, Tech. Rep. CASL-U-2012-0131-003, Consortium for Advanced Simulation of LWRs (2014).

[19] A. T. Godfrey, VERA core physics benchmark progression problem specifications, Tech. Rep. CASL-U-2014-0045-000, Consortium for Advanced Simulation of LWRs (2014).

[20] K. S. Kim, M. L. Williams, D. Wiarda, A. T. Godfrey, VERA core physics benchmark progression problem specifications, Tech. Rep. CASLU-2015-0180-000, Consortium for Advanced Simulation of LWRs (2015).

[21] J. Gehin, A. Godfrey, F. Franceschini, T. Evans, B. Collins, S. Hamilton, Operational reactor model demonstration with VERA: Watts Bar unit 1 cycle 1 zero power physics tests, Tech. Rep. CASL-U-2013-0105-001, Consortium for Advanced Simulation of LWRs (2013).

[22] Oak Ridge Leadership Computing Facility, Titan Cray XK7 (January 2015).

URL https://www. olcf .ornl.gov/computing-resources/titan-cray-xk7/

[23] F. Franceschini, A. Godfrey, J. Kulesza, R. Oelrich, Westinghouse VERA test stand - zero power physics test simulations for the AP1000 PWR, Tech. Rep. CASL-U-2014-0012-000, Consortium for Advanced Simulation of LWRs (2014). 